\title{
ON THE EXISTENCE OF PRIME IDEALS IN BOOLEAN ALGEBRAS
}

\author{
JÖRG FLUM \\ Mathematisches Institut, Universität Freiburg \\ Eckerstr. 1 \\ 79104 Freiburg, Germany \\ E-mail: flum@ruf.uni-freiburg.de
}

\begin{abstract}
Rasiowa and Sikorski [5] showed that in any Boolean algebra there is an ultrafilter preserving countably many given infima. In [3] we proved an extension of this fact and gave some applications. Here, besides further remarks, we present some of these results in a more general setting.
\end{abstract}

1. Introduction. Let $E$ be a subset and $a$ an element of a Boolean algebra $\mathcal{B}, E \subseteq B$ and $a \in B$. Assume that $a$ is the infimum of $E, a=\bigwedge E$. An ultrafilter $U$ preserves $a=\bigwedge E$, if

$$
a \notin U \text { implies } e \notin U \text { for some } e \in E \text {. }
$$

In the section entitled "A theorem on the existence of prime ideals in Boolean algebras" of their paper "A proof of the completeness theorem of Gödel" (cf. [5]), Rasiowa and Sikorski prove the following theorem which is sometimes (cf. [4]) called the Lemma of Rasiowa and Sikorski.

Theorem 1.1. Given infima $a_{1}=\bigwedge E_{1}, a_{2}=\bigwedge E_{2}, \ldots$ in a non-trivial (i.e., $0 \neq 1$ ) Boolean algebra there is an ultrafilter preserving all these infima.

Since

$$
a=\bigwedge E \quad \text { implies } \quad 0=\bigwedge\{e \cap \sim a \mid e \in E\}
$$

this result can be rephrased as:

COROllary 1.2. Let $E_{1}, E_{2}, \ldots$ be subsets of a non-trivial Boolean algebra with $0=$ $\bigwedge E_{1}=\bigwedge E_{2}=\ldots$ Then

(*) there is an ultrafilter $U$ s.t. for all $n$ there is $e \in E_{n}$ with $\sim e \in U$.

1991 Mathematics Subject Classification: 03G05, 03E50, 06E10, 54D80.

The paper is in final form and no version of it will be published elsewhere. 
In [3] we gave necessary and sufficient "absolute" conditions for the existence of an ultrafilter as in this corollary in case we omit the hypothesis $0=\bigwedge E_{1}=\bigwedge E_{2}=\ldots$. (As shown by $E_{1}=\{a\}$ and $E_{2}=\{\sim a\}$ with an arbitrary element $a$ the hypothesis cannot simply be omitted.)

Our result and proof method were inspired by a corresponding characterization of the omissible types of (incomplete) first-order theories contained in [1], rediscovered and applied in [2]. It is well-known that one of the first important applications of the Lemma of Rasiowa and Sikorski is its use by Ryll-Nardzewski to characterize $\omega_{0}$-categorical theories (cf. [6]). Implicitly, this characterization contains the so-called omitting types theorem.

In this paper we present our results extending the Lemma of Rasiowa and Sikorski in a more general setting.

2. Inflationary and monotone operations. Let $B$ be a set and $J$ an operation on ther power set of $B$,

$$
J: \operatorname{Pow}(B) \rightarrow \operatorname{Pow}(B),
$$

that is inflationary and monotone; here inflationary means that

$$
X \subseteq J(X),
$$

and monotone that

$$
X \subseteq Y \text { implies } J(X) \subseteq J(Y) .
$$

By transfinite induction one defines the subsets $J_{\alpha}$ of $B$ by

$$
J_{0}:=\emptyset ; \quad J_{\alpha+1}:=J\left(J_{\alpha}\right) ; \quad J_{\alpha}:=\bigcup_{\beta<\alpha} J_{\beta}
$$

Then,

is the least fixed-point of $J$, i.e.,

$$
J_{\infty}:=\bigcup_{\alpha} J_{\alpha}
$$

$$
J\left(J_{\infty}\right)=J_{\infty} \text { and } J(X)=X \text { implies } J_{\infty} \subseteq X .
$$

If $\kappa$ is an infinite cardinal, we say that $J$ is $\kappa$-ary, if

$$
J(X)=\bigcup\left\{J\left(X_{0}\right) \mid X_{0} \subseteq X \text { and }\left|X_{0}\right|<\kappa\right\}
$$

(here $|Y|$ denotes the cardinality of $Y$ ).

Now let $I$ be a set and for $i \in I$ let $J^{i}$ be an inflationary and monotone operation on the power set of $B$. Define the union of $J^{I}$ of the $J^{i}$, s,

$$
J^{I}: \operatorname{Pow}(B) \rightarrow \operatorname{Pow}(B),
$$

by

$$
J^{I}(X):=\bigcup\left\{J^{i}(X) \mid i \in I\right\}
$$

Clearly, $J^{I}$ is inflationary and monotone. Moreover,

(1) Every fixed-point of $J^{I}$ is a fixed-point of each $J^{i}$; in particular, $J_{\infty}^{I}$ is a fixed-point of each $J^{i}$. 
Proof. Assume that $J^{I}(X)=X$. Since $J^{i}$ is inflationary, we have $X \subseteq J^{i}(X) \subseteq$ $J^{I}(X)=X$.

(2) If each $J^{i}$ is $\kappa$-ary, then so is $J^{I}$ and, for any $\alpha$,

$$
J_{\alpha}^{I}=\bigcup\left\{J_{\alpha}^{I_{0}} \mid I_{0} \subseteq I \text { and }\left|I_{0}\right|<\kappa\right\}
$$

(here, $J^{I_{0}}$ is the union of the $J^{i}$ 's with $i \in I_{0}$ ).

Proof. Clearly, the equality holds for $\alpha=0$. For $\alpha=\beta+1$ we have

$$
\begin{aligned}
J_{\beta+1}^{I} & :=J^{I}\left(J_{\beta}^{I}\right)=\bigcup_{i \in I} J^{i}\left(J_{\beta}^{I}\right) \\
& =\bigcup_{i \in I} \bigcup_{I_{0} \subseteq I,\left|I_{0}\right|<\kappa} J^{i}\left(J_{\beta}^{I_{0}}\right) \\
& =\bigcup_{I_{0} \subseteq I,\left|I_{0}\right|<\kappa} J^{I_{0}}\left(J_{\beta}^{I_{0}}\right)=\bigcup_{I_{0} \subseteq I,\left|I_{0}\right|<\kappa} J_{\beta+1}^{I_{0}}
\end{aligned}
$$

(in deriving the first equality in the last line note that $I_{1} \subseteq I_{2}$ implies $J^{I_{1}}(X) \subseteq J^{I_{2}}(X)$ ). If $\alpha$ a limit ordinal then

$$
J_{\alpha}^{I}=\bigcup_{\beta<\alpha} J_{\beta}^{I}=\bigcup_{I_{0} \subseteq I,\left|I_{0}\right|<\kappa} \bigcup_{\beta<\alpha} J_{\beta}^{I_{0}}=\bigcup_{I_{0} \subseteq I,\left|I_{0}\right|<\kappa} J_{\alpha}^{I_{0}} .
$$

As a corollary we get:

(3) If each $J^{i}$ is $\kappa$-ary, then $J_{\infty}^{I}=\bigcup\left\{J_{\infty}^{I_{0}} \mid I_{0} \subseteq I\right.$ and $\left.\left|I_{0}\right|<\kappa\right\}$.

Hence,

(4) If each $J^{i}$ is $\kappa$-ary, then for $a \in B$,

$$
a \in J_{\infty}^{I} \quad \text { iff } \quad a \in J_{\infty}^{I_{0}} \text { for some } I_{0} \subseteq I \text { with }\left|I_{0}\right|<\kappa .
$$

3. The generalization of the Lemma of Rasiowa and Sikorski. Fix a Boolean algebra $\mathcal{B}$. For a subset $X$ of $B$ denote by $F(X)$ the filter generated by $X$,

$$
F(X):=\left\{b \mid \text { there are } n \geq 0, a_{0}, \ldots, a_{n} \in X \text { with } a_{0} \cap \ldots \cap a_{n} \leq b\right\} .
$$

A filter $F$ is proper, if $0 \notin F$. Henceforth, we shall use the letter $U$ to denote ultrafilters, i.e., proper filters such that $a \in U$ or $\sim a \in U$ for all $a \in B$.

An ultrafilter $U$ omits $E$, if there is $e \in E$ such that $\sim e \in U$ (cf. 1.2). Then, we say that $E$ is omissible. Define $J\left(=J^{E}\right), J: \operatorname{Pow}(B) \rightarrow \operatorname{Pow}(B)$, by

$$
J(X):=\{\sim a \mid E \subseteq F(X \cup\{a\})\} .
$$

Clearly,

(5) $J$ is inflationary and monotone; if $|E|<\kappa$ then $J$ is $\kappa$-ary.

(6) If $X \subseteq U$ and $U$ omits $E$, then $J(X) \subseteq U$.

Proof. Assume $X \subseteq U, U$ omits $E$, and let $\sim a \in J(X)$. Then, $E \subseteq F(X \cup\{a\}) \subseteq$ $F(U \cup\{a\})$. Therefore, $\sim a \in U$

A simple transfinite induction using (6) shows

(7) if $U$ omits $E$ then $J_{\infty} \subseteq U$. 
Moreover,

(8) $J(X)=X$ iff $X$ is a filter and in the quotient Boolean algebra $\mathcal{B} / X$ we have $\bigwedge \bar{E}=0$

(here $\bar{E}=\{\bar{e} \mid e \in E\}$, where $\bar{e}$ denotes the equivalence class of $e$ ).

Proof. First, assume the right side of the equivalence. We only must show that $J(X) \subseteq X$. So assume $b \in J(X)$. Then $E \subseteq F(X \cup\{\sim b\})$. Since $\bigwedge \bar{E}=0$ in $\mathcal{B} / X$, we have $\overline{\sim b}=0$ in $\mathcal{B} / X$, thus $b \in X$.

Now assume $J(X)=X$. Let $x \in X$. Then, $E \subseteq F(X \cup\{\sim x\})$. If $y \in X$ then $F(X \cup\{\sim$ $x\})=F(X \cup\{\sim x \cup \sim y\})$, hence, $E \subseteq F(X \cup\{\sim(x \cap y)\})$, thus $x \cap y \in J(X)=X$. If $x \leq y$ then $F(X \cup\{\sim y\}) \supseteq F(X \cup\{\sim x\}) \supseteq E$ and therefore, $y \in J(X)=X$. Finally, let $a \in B$, and assume that in $\mathcal{B} / X$,

$\bar{a} \leq \bar{e}$ for all $e \in E$.

Then $E \subseteq F(X \cup\{a\})$, thus, $\sim a \in J(X)=X$, hence, $\bar{a}=0$.

Now let $\mathcal{E}$ be a non-empty class of subsets of $B$. We say that $\mathcal{E}$ is onissible, if there is an ultrafilter $U$ that omits $\mathcal{E}$, i.e., that omits each $E$ in $\mathcal{E}$. Let $J^{\mathcal{E}}$ be the union of the $J^{E}$ 's for $E \in \mathcal{E}$, i.e.,

$$
J^{\mathcal{E}}(X)=\bigcup_{E \in \mathcal{E}} J^{E}(X)=\{\sim a \mid E \subseteq F(X \cup\{a\}) \text { for some } E \in \mathcal{E}\} .
$$

A transfinite induction, using (7), shows:

(9) If $U$ omits $\mathcal{E}$ then $J_{\infty}^{\mathcal{E}} \subseteq U$.

By (1) and (8) we get

(10) $J_{\infty}^{\mathcal{E}}$ is a filter and in the quotient Boolean algebra $\mathcal{B} / J_{\infty}^{\mathcal{E}}$ we have $\bigwedge \bar{E}=0$ for every $E \in \mathcal{E}$

Let $\mathcal{C}$ be a class of Boolean algebras and $\lambda$ a cardinal. We say that $\mathcal{C}$ is $\mathrm{R}($ asiowa) $\mathrm{S}($ ikorski) $(\lambda)$-good, if for any non-trivial Boolean algebra $\mathcal{B}$ in $\mathcal{C}$ and any set $\mathcal{E},|\mathcal{E}|<\lambda$, of non-empty subsets $E$ of $B$ with $\bigwedge E=0$, there is an ultrafilter $U$ that omits $\mathcal{E}$. The classical Lemma of Rasiowa and Sikorski (cf. 1.2) tells us that the class of all Boolean algebras is $\mathrm{RS}\left(\omega_{1}\right)$-good. Martin's axiom is (equivalent to) the statement that the class of all Boolean algebras with the countable chain condition is $\operatorname{RS}\left(2^{\omega}\right)$-good (a Boolean algebra satisfies the countable chain condition, if every subset of pairwise disjoint elements is countable). The class of all Boolean algebras is not $\mathrm{RS}\left(\omega_{1}^{+}\right)$-good; a counterexample is obtained by choosing an appropriate set $\mathcal{E}$ in the Boolean algebra of regular open subsets of the partial order given by the set of partial functions from $\omega$ to $\omega_{1}$ with finite support (cf. [4]).

TheOREM 3.1. Let $\mathcal{C}$ be a $\mathrm{RS}(\lambda)$-good class of Boolean algebras closed under quotients. Then, for any Boolean algebra $\mathcal{B}$ in $\mathcal{C}$ and any family $\mathcal{E},|\mathcal{E}|<\lambda$, of subsets of $B$,

$$
\mathcal{E} \text { is omissible iff } 0 \notin J_{\infty}^{\mathcal{E}} .
$$

Proof. If $U$ omits $\mathcal{E}$, then $J_{\infty}^{\mathcal{E}} \subseteq U$ by (9); hence, $0 \notin J_{\infty}^{\mathcal{E}}$. Otherwise, if $0 \notin J_{\infty}^{\mathcal{E}}$ then, by $(8), J_{\infty}^{\mathcal{E}}$ is a proper filter, $\mathcal{B} / J_{\infty}^{\mathcal{E}}$ is a non-trivial Boolean algebra, and, in $\mathcal{B} / J_{\infty}^{\mathcal{E}}$, 
we have $\bigwedge \bar{E}=0$ for all $E \in \mathcal{E}$. Hence, by the assumption of $\operatorname{RS}(\lambda)$-goodness there is an ultrafilter $U$ in $\mathcal{B} / J_{\infty}^{\mathcal{E}}$ that omits $\{\bar{E} \mid E \in \mathcal{E}\}$. Therefore, $U^{-1}:=\{b \in B \mid \bar{b} \in U\}$ is an ultrafilter omitting $\mathcal{E}$.

Recall that a Boolean algebra $\mathcal{B}$ is retractive, if for every proper filter $F$ in $\mathcal{B}$ there is a homomorphism $f$ from $\mathcal{B} / F$ to $\mathcal{B}$ such that $\pi \circ f$ is the identity on $\mathcal{B} / F$ (here, $\pi$ denotes the canonical homomorphism from $\mathcal{B}$ onto $\mathcal{B} / F)$. Clearly,

if $\mathcal{B}$ is retractive and has the ccc, then every quotient of $\mathcal{B}$ has the ccc.

Every interval algebra and every tree algebra is retractive (see [4]). Hence, we obtain from the preceding theorem (taking as $\mathcal{C}$ the class of interval algebras (or, the class of tree algebras) with ccc):

Corollary 3.2. Assume Martin's axiom and let $\mathcal{B}$ be an interval algebra or a tree algebra with the countable chain condition. Furthermore, let $\mathcal{E},|\mathcal{E}|<2^{\omega}$, be a family of subsets of $\mathcal{B}$. Then $\mathcal{E}$ is omissible iff $0 \notin J_{\infty}^{\mathcal{E}}$.

THEOREM 3.3. Let $\mathcal{C}$ be a $\mathrm{RS}(\lambda)$-good class of Boolean algebras closed under quotients. For $\mathcal{B}$ in $\mathcal{C}$ and any family $\mathcal{E},|\mathcal{E}|<\lambda$, of subsets $E$ of $\mathcal{B}$ with $|E|<\kappa$ the following holds: if every subfamily of $\mathcal{E}$ of cardinality less than $\kappa$ is omissible, then $\mathcal{E}$ is omissible.

Proof. Let $\mathcal{E}_{0}$ be an arbitrary subfamily of $\mathcal{E}$ of cardinality less than $\kappa$. Since $\mathcal{E}_{0}$ is omissible, $0 \notin J_{\infty}^{\mathcal{E}_{0}}$ by (9). As $J^{\mathcal{E}}$ is $\kappa$-ary (cf. (5) and (2)), we have by (3), $0 \notin J_{\infty}^{\mathcal{E}}$. Hence, by the previous theorem, $\mathcal{E}$ is omissible.

An instance of this theorem is:

Corollary 3.4. Assume Martin's axiom and let $\mathcal{E},|\mathcal{E}|<2^{\omega}$, be a family of countable subsets of an interval algebra or of a tree algebra with the countable chain condition. If every countable subfamily of $\mathcal{E}$ is omissible, then $\mathcal{E}$ is omissible.

\section{References}

[1] J. Barwise and Y. N. Moschovakis, Global inductive definability, Jour. Symb. Logic 43(1978), 521-534.

[2] E. Casanovas and R. Farré, Omitting types in incomplete theories, Jour. Symb. Logic 41(1996), 236-245.

[3] J. Flum, An extension of the lemma of Rasiowa and Sikorski, to appear.

[4] S. Koppelberg, General theory of Boolean algebras, Volume I of D. Monk, editor, Handbook of Boolean algebras, North-Holland, 1989.

[5] H. Rasiowa and R. Sikorski, A proof of the completeness theorem of Gödel, Fund. Math. 37(1950), 193-200.

[6] C. Ryll-Nardzewski, On the categoricity in power $\leq \omega$, Bull. Acad. Pol. Sci. 7(1959), $545-548$ 